\title{
Training linear ranking SVMs in linearithmic time using red-black trees
}

\author{
Antti Airola, Tapio Pahikkala and Tapio Salakoski
}

\begin{abstract}
We introduce an efficient method for training the linear ranking support vector machine. The method combines cutting plane optimization with red-black tree based approach to subgradient calculations, and has $O(m s+m \log (m))$ time complexity, where $m$ is the number of training examples, and $s$ the average number of non-zero features per example. Best previously known training algorithms achieve the same efficiency only for restricted special cases, whereas the proposed approach allows any real valued utility scores in the training data. Experiments demonstrate the superior scalability of the proposed approach, when compared to the fastest existing RankSVM implementations.
\end{abstract}

\section{Introduction}

Learning to rank has been a task of significant interest during the recent years. The ranking problem has been largely motivated by applications in areas such as web search and recommender systems. Due to the large amounts of data available in these domains, it is necessary for the used algorithms to scale well, preferably close to linear time methods are needed. For a detailed introduction to the topic of learning to rank, we refer to (Liu, 2009, Fürnkranz and Hüllermeier, 2011).

In this work we assume the so-called scoring setting, where each data instance is associated with a utility score reflecting its goodness with respect to the ranking criterion. A successful approach for learning ranking functions has been to consider pairwise preferences (Fürnkranz and Hüllermeier, 2005). In this setting, the aim is to minimize the number of pairwise mis-orderings in the ranking produced when ordering a set of examples according to predicted utility scores. A number of machine learning algorithms optimizing relaxations of this criterion have been proposed, such as the RankBoost (Freund et al., 2003), RankNet (Burges et al., 2005), RankRLS (Pahikkala et al., 2007, 2009), and the subject of this study, the ranking support vector machine (RankSVM) algorithm (Herbrich et al., 1999: Joachims, 2002).

The original solution proposed for RankSVM optimization was to train a support vector machine (SVM) classifier on pairs of data examples. Adapting standard dual SVM solvers to RankSVM training leads to $O\left(m^{4}\right)$ scaling or 
worse, with respect to the training set size $m$ (Bottou and Lin, 2007). While linear RankSVMs can be trained more efficiently by solving the primal optimization problem for SVMs (Chapelle, 2007; Chapelle and Keerthi, 2010), still the complexity of any method that is explicitly trained on all the pairs has at the very least quadratic dependence on the number of training examples.

Joachims (2005, 2006) has shown that linear RankSVM can be trained using cutting plane optimization, also known as bundle optimization, much more efficiently in certain special settings. Joachims (2005) has proposed an $O(m s+m \log (m))$ time algorithm, where $m$ is the number of training examples, and $s$ the average number of non-zero features per example, for the bipartite ranking problem, where only two utility levels are allowed. The bipartite ranking problem corresponds to maximizing the area under the receiving operating characteristic curve (AUC) (Hanley and McNeil, 1982), a performance measure widely used in machine learning (see e.g. (Bradley, 1997, Provost et al., 1998)). An $O(m s+m \log (m)+r m)$ time generalization of the method has been presented for the case, where $r$ different utility levels are allowed (Joachims, 2006). Chapelle and Keerthi (2010) have recently further explored efficient methods for training RankSVM, the proposed methods have similar scaling.

If $r$ is assumed to be a small constant, the existing methods are computationally efficient. This is for example the case in the bipartite ranking case where there are only two utility levels, corresponding to the "good" and the "bad" objects. Similarly, movie ratings ranging from one to five stars could be encoded using $r=5$ distinct utility levels. However, in the general case where unrestricted scores are allowed, if most of the training examples have different scores $r \approx m$ leading to $O\left(m s+m^{2}\right)$ complexity. This worst scale quadratic scaling with respect to the training set size limits the applicability of RankSVM to large scale learning.

In this work we generalize the work of Joachims (2006) and present an $O(m s+m \log (m))$ time training algorithm for linear RankSVM, that is applicable in the most general case, where arbitrary real-valued utility scores are allowed in the training data. The method is based on using modified red-black tree data structures (Bayer, 1972, Cormen et al., 2001) for speeding up the evaluations needed in the optimization process. Computational experiments show that the method has excellent scalability properties also in practice.

In Section 2 we introduce the general learning to rank setting, and in Section 3 we formalize the regularized risk minimization problem, and present the general optimization framework for solving it. In Section 4 we present our main contribution, the efficient RankSVM subgradient and loss computation algorithms, and in Section 5 we present an experimental evaluation of the resulting training algorithm. We conclude in Section 6 .

\section{Learning setting}

Let $D$ be a probability distribution over a sample space $\mathcal{Z}=\mathbb{R}^{n} \times \mathbb{R}$. An example $z=(\mathbf{x}, y) \in \mathcal{Z}$ is a pair consisting of an $n$-dimensional column vector of real- 
valued features, and an associated real-valued utility score. Let the sequence $Z=\left(\left(\mathbf{x}_{1}, y_{1}\right), \ldots,\left(\mathbf{x}_{m}, y_{m}\right)\right) \in \mathcal{Z}^{m}$ drawn according to $D$ be a training set of $m$ training examples. $X \in \mathbb{R}^{n \times m}$ denotes the $n \times m$ data matrix whose columns contain the feature representations of the training examples, and $\mathbf{y} \in \mathbb{R}^{m}$ is a column vector containing the utility scores in the training set. Our task is to learn from the training data a ranking function $f: \mathbb{R}^{n} \rightarrow \mathbb{R}$. In the linear case such a function can be represented as $f(\mathbf{x})=\mathbf{w}^{\mathrm{T}} \mathbf{x}$, where $\mathbf{w} \in \mathbb{R}^{n}$ is a vector of parameters.

The difference between ranking and regression is that in ranking, the actual values taken by the prediction function are typically not of interest. Rather, what is of interest is how well the ordering acquired by sorting a set of new examples according to their predicted scores matches the true underlying ranking. This is a reasonable criterion for example in the web search engines and recommender systems, where the task is to choose a suitable order in which to present web pages or products to the end user. A popular way to model this criterion is by considering the pairwise preferences induced by a ranking (see e.g. (Fürnkranz and Hüllermeier, 2005)). We say that an example $z_{i}$ is preferred over example $z_{j}$, if $y_{i}>y_{j}$. In this case one would require from the ranking function that $f\left(\mathbf{x}_{i}\right)>f\left(\mathbf{x}_{j}\right)$.

The performance of a ranking function can be measured by the pairwise ranking error defined as

$$
\frac{1}{N} \sum_{y_{i}<y_{j}}\left[f\left(\mathbf{x}_{i}\right)>f\left(\mathbf{x}_{j}\right)\right],
$$

where $N$ is the number of pairs for which $y_{i}<y_{j}$ holds true. The equation (1) simply counts the number of swapped pairs between the true ranking and the one produced by $f$.

By restricting the allowed range of utility scores we can recover some popular special cases of the introduced setting. In ordinal regression (Herbrich et al. 1999, Waegeman et al., 2008) it is assumed that there exists a finite, often quite small set of possible discrete ordinal labels. For example, movie ratings ranging from one star to five stars would constitute such a scale. In the bipartite ranking task where only two possible scores are allowed equation (1) becomes equivalent to the Wilcoxon-Mann-Whitney formula used to calculate AUC (Hanley and McNeil, 1982, Cortes and Mohri, 2004).

In some learning to rank settings instead of having a total order over all examples, the sample space is divided into disjoint subsets, and pairwise preferences are induced only from pairwise comparisons between the scores of examples in the same subset. An example of an application settings where this approach is commonly adopted is document retrieval, where data consists of query-document pairs, and the scores represent the utility of the document with respect to the associated user query (Joachims, 2002). Preferences are induced only between query-document pairs from the same query, never between examples from different queries. In such settings we can calculate (1) separately for each subset, and take the average value as the final error. 
Minimizing (1) directly is computationally intractable, successful approaches to learning to rank according to the pairwise criterion typically minimize convex relaxations instead. The relaxation considered in this work is the pairwise hinge loss, which together with a quadratic regularizer forms the objective function of RankSVM. Before formally defining the loss, we introduce a general optimization method suitable for minimizing it.

\section{Bundle Methods for Regularized Risk Mini- mization}

A large class of machine learning algorithms can be formulated as the unconstrained regularized risk minimization problem

$$
\mathbf{w}^{*}=\underset{\mathbf{w} \in \mathbb{R}^{n}}{\arg \min } J(\mathbf{w}),
$$

where

$$
J(\mathbf{w})=R_{e m p}(\mathbf{w})+\lambda\|\mathbf{w}\|^{2},
$$

$\mathbf{w}$ is the vector of parameters to be learned, $R_{e m p}$ is the empirical risk measuring how well $\mathbf{w}$ fits the training data, $\|\mathbf{w}\|^{2}$ is the quadratic regularizer measuring the complexity of the considered hypothesis, and $\lambda \in \mathbb{R}^{+}$is a parameter. We assume that $R_{e m p}: \mathbb{R}^{n} \rightarrow \mathbb{R}$ is convex and non-negative.

Different choices of $R_{e m p}$ result in different machine learning methods such as SVM classification (Cortes and Vapnik, 1995) or regression (Drucker et al. 1997), regularized least-squares regression (Poggio and Girosi, 1990), structured output prediction methods (Tsochantaridis et al., 2005), RankRLS (Pahikkala et al. 2007, 2009) and RankSVM (Herbrich et al. 1999, Joachims, 2002).

Bundle methods for regularized risk minimization (BMRM) (Teo et al. 2007. Smola et al., 2007; Teo et al., 2010), is a general and efficient optimization technique for solving (2). The method is also known as the cutting plane method in the machine learning literature, and it was under this name it was first introduced for the purpose of efficiently optimizing large margin type of loss functions (Tsochantaridis et al. 2005). It was later shown, that the method can be generalized to arbitrary convex loss functions, as long as subgradient evaluations for the loss can be done efficiently (Teo et al., 2007, Smola et al. 2007). In the following we adopt our notation and terminology from the BMRM literature.

BMRM iteratively constructs a piecewise linear lower bound approximation of $R_{e m p}$. Let $R_{t}$ be the piecewise linear approximation at iteration $t$. We approximate 2 with

$$
\mathbf{w}_{t}=\underset{\mathbf{w} \in \mathbb{R}^{n}}{\arg \min } J_{t}(\mathbf{w})
$$

where

$$
J_{t}(\mathbf{w})=R_{t}(\mathbf{w})+\lambda\|\mathbf{w}\|^{2}
$$


Thus the regularizer remains the same, but the empirical risk term is replaced with the piecewise linear lower bound.

The empirical risk is lower bounded by its first order Taylor approximation at any $\mathbf{w}^{\prime} \in \mathbb{R}^{n}$, defined as

$$
R_{e m p}(\mathbf{w}) \geq R_{e m p}\left(\mathbf{w}^{\prime}\right)+\left\langle\mathbf{w}-\mathbf{w}^{\prime}, \mathbf{a}^{\prime}\right\rangle,
$$

where $\mathbf{a}^{\prime}$ is any subgradient of $R_{e m p}$ at $\mathbf{w}^{\prime}$. By defining $b^{\prime}=R_{e m p}\left(\mathbf{w}^{\prime}\right)-\left\langle\mathbf{w}^{\prime}, \mathbf{a}^{\prime}\right\rangle$ due to the linearity of the inner product this can be re-written as

$$
R_{e m p}(\mathbf{w}) \geq\left\langle\mathbf{w}, \mathbf{a}^{\prime}\right\rangle+b^{\prime}
$$

$\left\langle\mathbf{w}, \mathbf{a}^{\prime}\right\rangle+b^{\prime}=0$ is called a cutting plane.

Using several cutting planes BMRM approximates $R_{e m p}$ with

$$
\left.R_{t}(\mathbf{w})=\max _{i=1 \ldots t}\left\{\left\langle\mathbf{w}, \mathbf{a}_{i}\right\rangle+b_{i}\right\}\right\} .
$$

Using this approximation (3) can be solved by solving an equivalent quadratic program whose size depends on the number of cutting planes used.

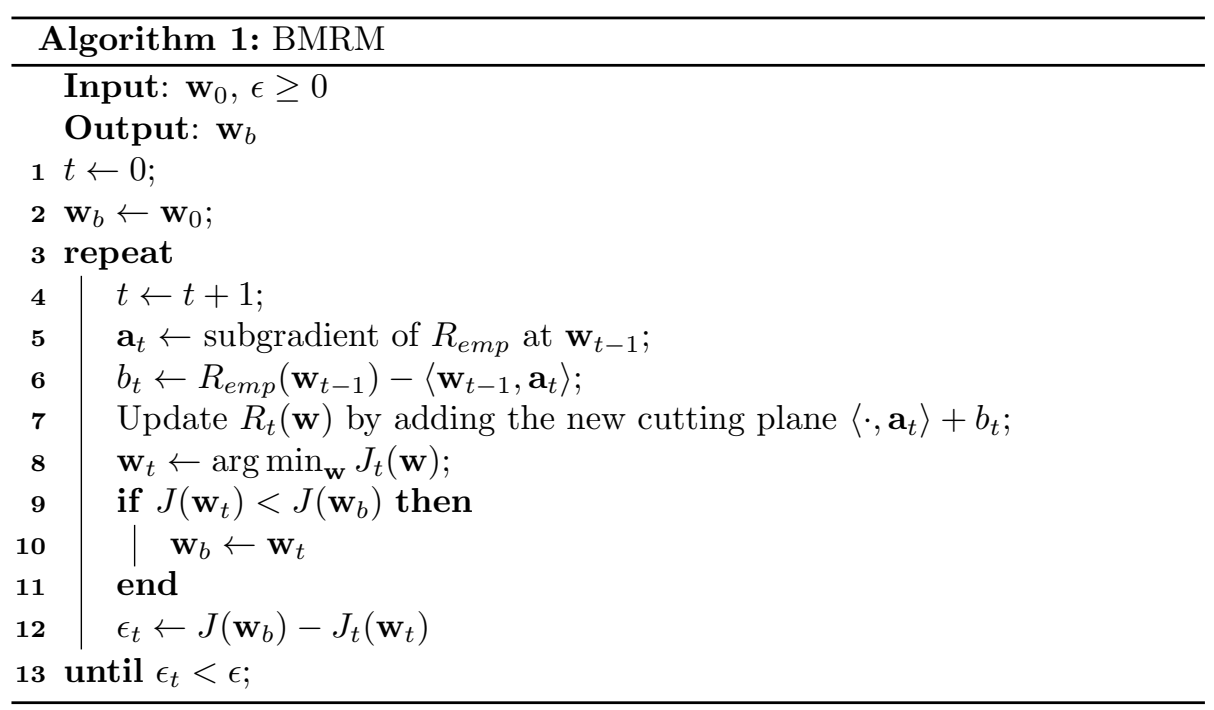

The procedure according to which BMRM builds the lower bound is described in algorithm 1. The formulation given here differs slightly from that of Teo et al. (2010) in that following the suggestion of Franc and Sonnenburg (2009) we maintain the best this far seen solution $\mathbf{w}_{b}$, and only update the solution when the new solution $\mathbf{w}_{t}$ is better. The parts of the algorithm which depend on the choice of $R_{e m p}$ are the calculation of a subgradient and the value of $R_{e m p}$ at point $\mathbf{w}_{t-1}$, as well as the termination criterion.

The rate of convergence is for BMRM independent of the training set size (Smola et al. 2007). The size of the quadratic program solved on line 8 does grow 
with the number of iterations, but its computational cost is on large datasets insignificant compared to the cost of computing the cutting plane. Thus what is required for efficient learning with a convex loss function is an efficient algorithm for computing its value and subgradient.

\section{Efficient computation of loss and subgradient}

Next we present an efficient algorithm for evaluating the empirical risk and its subgradient for RankSVM. First, in Section 4.1 we recall results from Joachims (2006) to identify the computational bottleneck in RankSVM training, which occurs in the loss and subgradient computation. Next, in Section 4.2 we introduce search tree algorithms, which we use to speed up these computations. Building on these results we present an efficient algorithm for loss and subgradient computation in Section 4.3 .

\subsection{Preliminaries}

The following results were first presented by Joachims (2006), who formulated the RankSVM optimization problem as a constrained optimization problem. In this work we follow a different but equivalent formulation of RankSVM as an unconstrained optimization problem within the BMRM framework (see Teo et al. (2010)).

The average pairwise hinge loss computed over the training set, with respect to a given solution $\mathbf{w}$ is

$$
R_{e m p}(\mathbf{w})=\frac{1}{N} \sum_{y_{i}<y_{j}} \max \left(0,1+\mathbf{w}^{\mathrm{T}} \mathbf{x}_{i}-\mathbf{w}^{\mathrm{T}} \mathbf{x}_{j}\right),
$$

where $N$ is the number of pairs for which $y_{i}<y_{j}$ holds true.

The pairwise hinge loss, together with the quadratic regularizer forms the objective function of RankSVM. The most obvious approach to evaluating (4), and also its subgradient involves going explicitly through all the pairs in the training set. However, this would lead to $O\left(\mathrm{~m}^{2}\right)$ complexity, which is inefficient on large data sets. Let us define

$$
c_{i}=\left|\left\{j:\left(y_{i}<y_{j}\right) \wedge\left(\mathbf{w}^{\mathrm{T}} \mathbf{x}_{i}>\mathbf{w}^{\mathrm{T}} \mathbf{x}_{j}-1\right) \wedge(1 \leq j \leq m)\right\}\right|
$$

and

$$
d_{i}=\left|\left\{j:\left(y_{i}>y_{j}\right) \wedge\left(\mathbf{w}^{\mathrm{T}} \mathbf{x}_{i}<\mathbf{w}^{\mathrm{T}} \mathbf{x}_{j}+1\right) \wedge(1 \leq j \leq m)\right\}\right| .
$$

Using the frequencies (5) and (6), we recover an alternative formulation for the empirical risk.

Lemma 1 (Joachims (2006); Teo et al. (2010)). The average pairwise hinge loss (4) can be equivalently expressed as

$$
\frac{1}{N} \sum_{i=1}^{m}\left(\left(c_{i}-d_{i}\right) \mathbf{w}^{T} \mathbf{x}_{i}+c_{i}\right) .
$$


Similarly, computation of (5) and (6) allows the computation of a subgradient of the empirical risk.

Lemma 2 (Joachims (2006); Teo et al. (2010)). A subgradient of (4) can be expressed as

$$
\nabla R_{e m p}(\mathbf{w})=\frac{1}{N} \sum_{i=1}^{m}\left(c_{i}-d_{i}\right) \mathbf{x}_{i} .
$$

Inner product evaluations, scalar-vector multiplications and vector summations are needed to calculate (7) and (8). These take each $O(s)$ time, the average number of nonzero elements in a feature vector. Provided that we know the values of $c_{i}, d_{i}$ and $N$, both the loss and subgradient can thus be evaluated in $O(m s)$ time.

Joachims (2006) (and equivalently Teo et al. (2010) ) describe a way to calculate efficiently these frequencies, and subsequently the loss and subgradient. However, the work assumes that the range of possible utility score values is restricted to $r$ different values, with $r$ being quite small. The algorithm requires $O(r)$ passes through the training set, contributing a $O(\mathrm{rm})$ term to the overall complexity, which is $O(m s+m \log (m)+r m)$. If the number of allowed scores is not restricted, at worst case $r=m$ with the resulting complexity $O\left(m s+m^{2}\right)$, meaning quadratic behavior with respect to the training set size. However, as we show next, the dependence on $r$ can be removed from the algorithm by utilizing order statistics trees, resulting in $O(m s+m \log (m))$ cost also in the most general case, where arbitrary real valued utility scores are allowed.

\subsection{Order statistics tree}

The order statistics tree (Cormen et al., 2001) is a balanced binary search tree, which has been augmented to support logarithmic time computation of order statistics, such as the rank of a given element in the tree, or the recovery of the $k$ :th element in the tree. As we will show in the following, the data structure also allows efficient computation of the frequencies needed in the RankSVM loss and subgradient computations. Next, we introduce the order statistics tree, recall its main properties, and introduce algorithms which act as building blocks for the fast RankSVM training method. In the following, we assume that the number of elements inserted to the search tree is bounded by $m$.

The binary search tree is one of the most fundamental data structures in computer science. It is a linked data structure consisting of nodes. A given node $x$ contains a real valued $\operatorname{key}(x)$, and pointers to a parent node $\operatorname{par}(x)$, a left child left $(x)$, and a right child right $(x)$. A parent or a child may be missing, denoted by the value $\emptyset$. Only a single node known as the root node is allowed to have missing parent. Nodes with no children are called leaf nodes. The root of an empty tree is assumed to be $\emptyset$. The height of a binary search tree is the length of the path from the root node to the lowest leaf node, and the size of a tree is the number of elements it contains. We call the tree rooted at left $(x)$ the left subtree of $x$, and the tree rooted at right $(x)$ the right subtree of $x$. The tree 
always satisfies the binary search tree property, requiring that the nodes stored in the left subtree of a node have smaller than or equal keys as the node, and the nodes in the right subtree have larger than or equal keys as the node.

The worst-case cost of searching for an element in the tree, or inserting or deleting an element is proportional to the height of the tree. To guarantee the efficiency of such operations, self-balancing binary trees combine basic tree update operations with maintenance operations that maintain $O(\log (m))$ tree height. One of the most popular self-balancing search tree variants is the redblack tree (Bayer, 1972; Cormen et al. 2001). Further, (Cormen et al., 2001) describe a modified variant of the red-black tree defined as follows:

Definition 1 (Order statistics tree (Cormen et al. (2001))). The order statistics tree is a self-balancing binary search tree, with the following properties

- The binary search tree property: "Let $x$ be a node in a binary search tree. If $y$ is a node in the left subtree of $x$, then $\operatorname{key}(y) \leq \operatorname{key}(x)$. If $y$ is a node in the right subtree of $x$, then $\operatorname{key}(x) \leq \operatorname{key}(y)$."

- Balance: $O(\log (m))$ height after arbitrary insertions and deletions.

- Each node of the order statistics tree stores an additional attribute size defined as

$$
\operatorname{size}(x)=\left\{\begin{array}{ll}
0 & \text { if } x=\emptyset \\
\operatorname{size}(\operatorname{left}(x))+\operatorname{size}(\operatorname{right}(x))+1 & \text { otherwise }
\end{array} .\right.
$$

The correct value for this attribute is maintained after arbitrary insertions and deletions.

Note that the definition allows the existence of multiple nodes with the same key value in the tree.

Let Tree-Insert $(T, x)$ be the insertion operation of an arbitrary node $x$ to an order statistics tree $T$, whose size is bounded by $m$. Tree-Insert adds the new node to the tree, maintaining the binary search tree property, the correct values of the size attribute in the nodes, and the $O(\log (m))$ height of the tree. The time complexity of the operation is characterized by the following Lemma:

Lemma 3 (Cormen et al. (2001)). The time complexity of Tree-Insert to order statistics tree is $O(\log (m))$.

Further, for the RankSVM computations we require routines that efficiently compute the number of elements in the tree with a smaller, or a larger value than a given argument. Let $k$ be a real value, and let $T$ be an order statistics tree, whose size is bounded by $m$. Then, Count-Smaller(root $(T), k$ ) (Algorithm 2 ) returns the number of nodes in $T$ with a smaller key value than $k$. The algorithm Count-Larger $(\operatorname{root}(T), k)$, which is not presented separately, works in analogous fashion returning the number of nodes with a larger key value than the argument.

Lemma 4. The correctness of Count-Smaller and Count-Larger. 
Proof. Let $C(x, k)$ denote the number of values smaller than $k$ stored in a binary search tree whose root $x$ is. Due to Definition 1, the following always holds

$$
C(x, k)= \begin{cases}0 & \text { if } x=\emptyset \\ C(\operatorname{right}(x), k)+\operatorname{size}(\operatorname{left}(x))+1 & \text { if key }(x)<k \\ C(\operatorname{left}(x), k) & \text { otherwise }\end{cases}
$$

This recursive equation supplies us directly with an algorithm for computing $C(x, k)$, which is implemented in Count-Smaller. The proof for Count-Larger is analogous.

Lemma 5. Count-Smaller and Count-Larger have $O(\log (m))$ complexity.

Proof. On each call of Count-Smaller or Count-Larger, $O(1)$ cost computations are performed, and additionally the routine may call itself once with a child node of the input node. At worst case the recursion proceeds until the lowest leaf node in the tree is reached, requiring a number of calls proportional to the height of the tree, which is according to Definition 1 guaranteed to be of the order $O(\log (m))$.

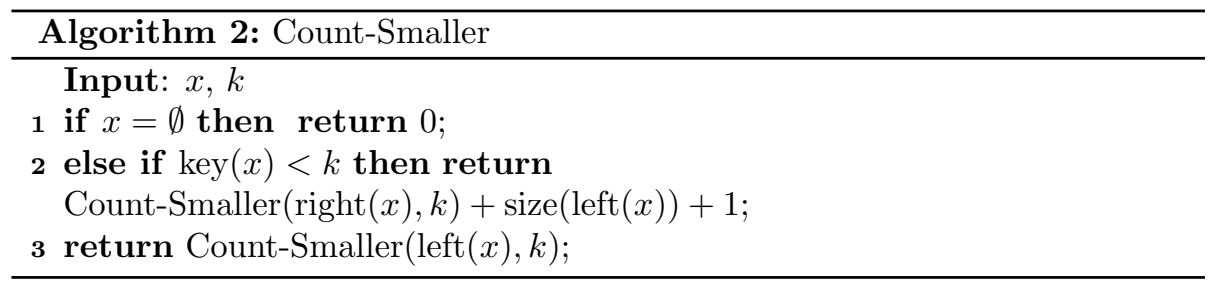

Let $r$ be the number of distinct keys stored in the tree. In the presence of a large number of duplicates, meaning $r<<m$, the order statistics tree can be implemented more efficiently by storing duplicate keys to the same node. In such an implementation, each node contains an additional attribute nodesize $(x)$, which measures how many times $\operatorname{key}(x)$ has been inserted to the tree. When inserting a new key, a new node is not created if the key already exists in the tree, but rather the nodesize attribute of the existing node is incremented by one. Thus, we need to re-define size $(x)=\operatorname{size}(\operatorname{left}(x))+\operatorname{size}(\operatorname{right}(x))+\operatorname{nodesize}(x)$. For this modified variant of the order statistics tree, the height of the tree, and the cost of Tree-Insert, Count-Smaller and Count-Larger are bounded by $O(\log (r))$. However, this improvement does not translate into further improvements in the asymptotic cost of RankSVM training, due to $O(m \log (m))$ cost of a sorting operation that is required on each iteration of training.

\subsection{Subgradient computation}

Algorithm 3 presents the main contribution of this paper, an $O(m s+m \log (m))$ time method for calculating the RankSVM loss and subgradient. The algorithm uses order statistics trees to efficiently compute the frequencies (5) and (6), 


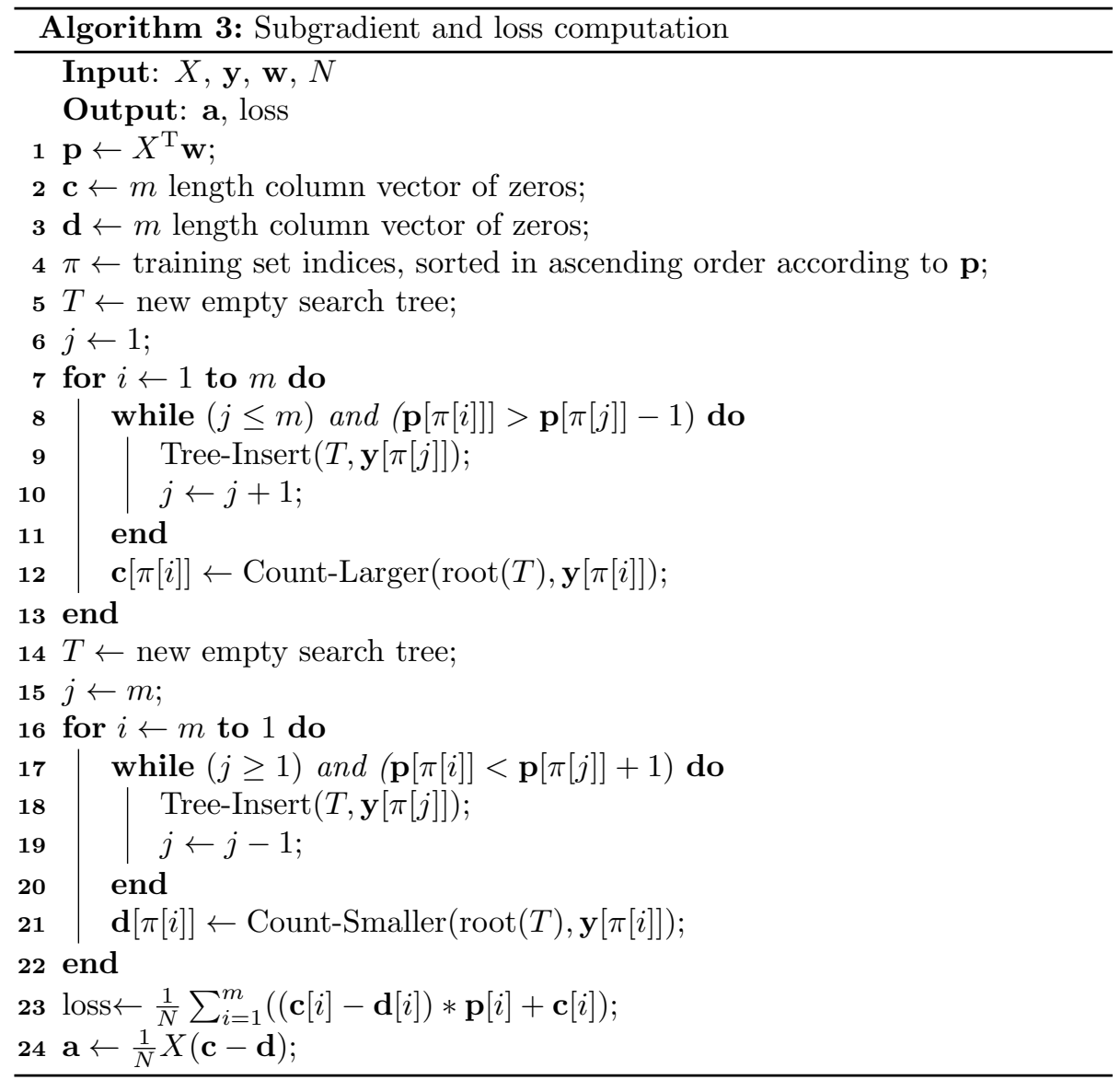


which are necessary in computing the value of the loss (7) and a subgradient (8).

Theorem 1. The correctness of Algorithm 3 .

Proof. The algorithm computes the predicted utility scores for the training set with $\mathbf{p}=X^{\mathrm{T}} \mathbf{w}$ (note that $\mathbf{p}[i]=\mathbf{w}^{\mathrm{T}} \mathbf{x}_{i}$ ). Next, an index list $\pi$ is created, where the indices of the training examples are ordered in an increasing order, according to the magnitudes of their predicted scores, so that $\mathbf{p}[\pi[1]] \leq \mathbf{p}[\pi[2]] \leq \ldots \leq$ $\mathbf{p}[\pi[m]]$.

Let us consider the $i$ :th iteration of the for-loop on lines $7-11$, with $1 \leq i \leq$ $m$. After the while loop on lines $8-10$ has been executed, the index $j$ divides the training set into two parts. For $1 \leq k<j$ it holds that $\mathbf{p}[\pi[i]]>\mathbf{p}[\pi[k]]-1$ and for $j \leq k \leq m$ it holds that $\mathbf{p}[\pi[i]] \leq \mathbf{p}[\pi[k]]-1$. The keys corresponding to the indices $\pi[1] \ldots \pi[j-1]$ have, either on this or on previous iterations, been inserted to the order statistics tree $T$, by the Tree-Insert call on line 9 . Therefore, on line 11 where Count-Larger $(\operatorname{root}(T), \mathbf{y}[\pi[i]])$ is called, $T$ contains the labels of the training examples indexed by the set

$$
\{k:(\mathbf{p}[\pi[i]]>\mathbf{p}[k]-1) \wedge(1 \leq k \leq m)\}
$$

According to Lemma 5, Count-Larger(root $(T), \mathbf{y}[\pi[i]])$ returns the number of keys in $T$, with a larger value than the argument $\mathbf{y}[\pi[i]]$. Thus, line 11 stores to $\mathbf{c}[\pi[i]]$ the value

$$
\left|\left\{k:\left(\mathbf{y}[\pi[i]]<y_{k}\right) \wedge(\mathbf{p}[\pi[i]]>\mathbf{p}[k]-1) \wedge(1 \leq k \leq m)\right\}\right|,
$$

which, according to (5) is the frequency $c_{\pi[i]}$.

It can be verified analogously, that the for-loop on lines $14-18$ computes the frequencies $d_{1} \ldots d_{m}$ according to (6). After line 18 has been executed, the algorithm has thus filled two arrays, $\mathbf{c}=\left[c_{1}, \ldots, c_{m}\right]$ and $\mathbf{d}=\left[d_{1}, \ldots, d_{m}\right]$. Using these frequencies, the loss is computed on line 19 according to (7), and the subgradient on line 20 according to (8).

Theorem 2. The complexity of calculating the loss and the subgradient with Algorithm 3 is $O(m s+m \log (m))$ for any training set of size $m$ and sparsity $s$, with unrestricted range for utility score values allowed.

Proof. The cost of computing $X^{\mathrm{T}} \mathbf{w}$ on line 1 using standard sparse matrix vector product multiplication algorithms is $O(\mathrm{~ms})$. Initializing the m-length arrays on lines 2 and 3 is a $O(m)$ operation, whereas the empty search tree initializations in lines 5 and 12 take $O(1)$ time. The sorting operation on line 4 can be done in $O(m \log (m))$ using for example the heapsort algorithm (Williams, 1964). Tree-Insert on line 9, and Count-Larger on line 11 are both called exactly $m$ times, once for each training example. According to Lemmas 3 and 5 both operations have $O(\log (m))$ cost, resulting in $O(m \log (m))$ complexity for the lines $7-11$ altogether. Analogously, lines $14-18$ have also $O(m \log (m))$ cost, 
since Tree-Insert on line 16 and the Count-Smaller on line 18 are both called exactly $m$ times. Finally, the loss computation on line 19 requires $O(m)$ floating point operations, and the subgradient computation on line 20 requires a $O(\mathrm{~ms})$ matrix-vector product. Summing all the complexities together, the resulting computational complexity of Algorithm 3 is $O(m s+m \log (m))$.

Next, we present a theorem that characterizes the overall complexity of RankSVM training using the introduced subgradient and loss computation algorithm. The theorem and its proof are similar to those presented by Joachims (2006). However, unlike Joachims, we do not (implicitly) assume the number of different relevance level to be constant.

Theorem 3. For any fixed $\epsilon>0$ and $\lambda>0$, the computational cost of linear RankSVM training with BMRM (Algorithm 1) using Algorithm 3 for loss and subgradient computations is $O(m s+m \log (m))$ for any training set of size $m$ and sparsity s, with unrestricted range for utility score values allowed.

Proof. Theorem 5 in (Smola et al. 2007) states that the BMRM has, under minor technical assumptions, $O\left(\frac{1}{\epsilon \lambda}\right)$ speed of convergence to $\epsilon$-accurate solution. The convergence speed does not depend on the values of $m$ and $s$. During initialization, the exact value of $N$ can be computed in $O(m \log (m))$ by sorting the true utility scores of the training examples. Further, the only computations within each iteration that depend on $m$ and $s$ are the loss and the subgradient computations. Therefore, the computational complexity of training RankSVM is the same as that of Algorithm 3 which is according to Theorem $2 \mathrm{O}(\mathrm{ms}+$ $m \log (m))$.

As discussed previously, in some ranking settings we do not have a global ranking over all examples. Instead, the training data may be divided into separate subsets, over each of which a ranking is defined. Let the training data set be divided into $R$ subsets, each consisting on average of $\frac{m}{R}$ examples. Then we can calculate the loss and the subgradient as the average over the losses and subgradients for each subset. The computational complexity becomes $O\left(R *\left(\frac{m}{R} s+\frac{m}{R} \log \left(\frac{m}{R}\right)\right)=O\left(m s+m \log \left(\frac{m}{R}\right)\right)\right.$.

\section{Computational experiments}

In the computational experiments we compare the scalability of the proposed $O(m s+m \log (m))$ time training algorithm to the fastest previously known approach. In addition, we compare our implementation to the existing publicly available RankSVM solvers. The considered data sets each contain a single global ranking, and the utility scores are real valued. This means that $r \approx m$, and the number of pairwise preferences in the training sets grows quadratically with $m$. In Section 5.1 we describe the experimental setup, and in Section 5.2 we present the experimental results. 

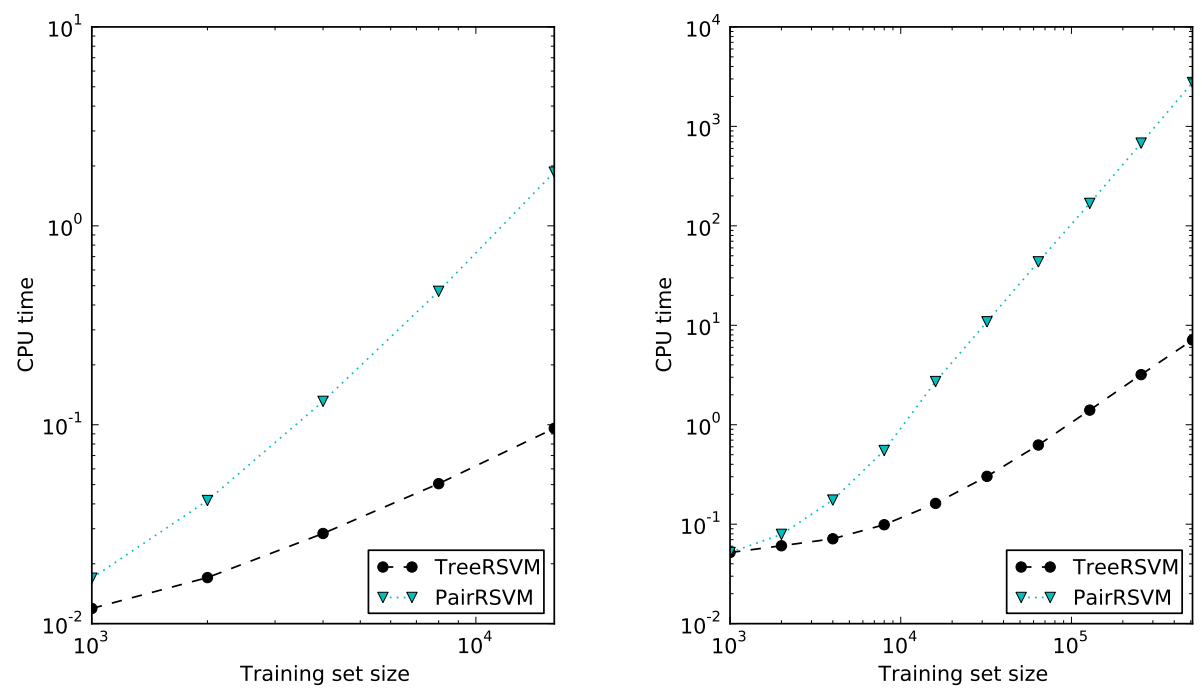

Figure 1: Average iteration cost. Cadata (left) and Reuters (right).

\section{$5.1 \quad$ Experimental setup}

We implement the proposed method, denoted as TreeRSVM, as well as a baseline method PairRSVM, which iterates over all pairs to calculate the frequencies necessary for the loss and subgradient computation. Both methods are based on our own implementation of BMRM, and are integrated to the RLScore 1 open source machine learning software framework developed by us. The majority of the code is written in Python. All matrix operations are implemented in NumPy and SciPy, and for solving the quadratic program arising in each BMRM iteration we use the CVXOPT ${ }^{2}$ open source convex optimization software. The most computationally demanding parts of the subgradient and loss computations, including the search tree implementation, are written in $\mathrm{C}$ language. The only difference between the two implementations is in the subgradient computation routine.

In addition, we compare our method to the fastest publicly available previous implementations of RankSVM. The SVM ${ }^{\text {rank }}$ software is a C-language implementation of the method described by Joachims (2006). In theory, SVM ${ }^{\text {rank }}$ and PairRSVM implement exactly the same method. In practice, implementational differences such as the use of different quadratic optimizers, and the inclusion of certain additional heuristics within $\mathrm{SVM}^{\text {rank }}$, mean that there may be some differences in their behavior. PRSVM implements in MATLAB a truncated Newton optimization based method for training RankSVM (Chapelle and

${ }^{1}$ http://www.tucs.fi/rlscore

${ }^{2}$ http://abel.ee.ucla.edu/cvxopt/ 

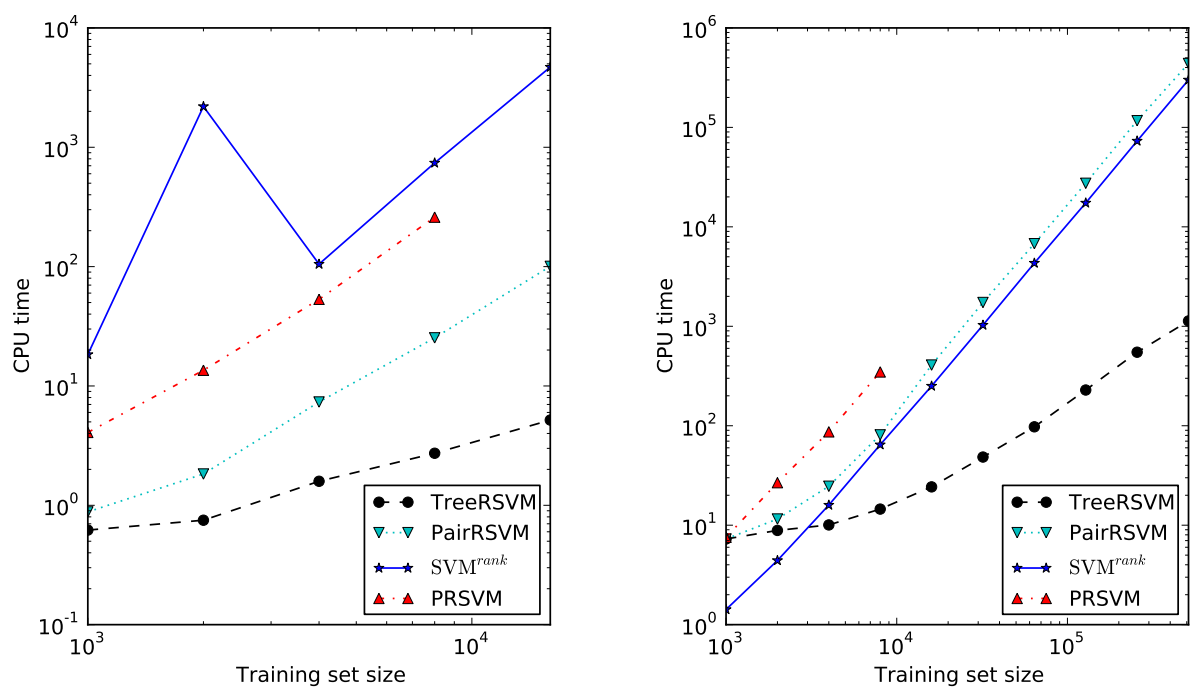

Figure 2: Runtimes for different RankSVM implementations. Cadata (left) and Reuters (right).

Keerthi, 2010). PRSVM optimizes a slightly different objective function than the other implementations, since it minimizes a squared version of the pairwise hinge loss. Finally, there exists an implementation of RankSVM in the SVM ${ }^{\text {light }}$ software package. It has however been previously shown to be orders of magnitude slower than either SVM ${ }^{\text {rank }}$ or PRSVM (Joachims, 2006, Chapelle and Keerthi, 2010), and therefore we do not include it in our comparison.

TreeRSVM has $O(m s+m \log (m))$ training time complexity, whereas all the other methods have $O\left(m s+m^{2}\right)$ training time complexity. Therefore, TreeRSVM should on large datasets scale substantially better than the other implementations. Further, all the methods other than PRSVM have $O(m s)$ memory complexity due to cost of storing the data matrix. PRSVM has $O\left(m s+m^{2}\right)$ memory complexity, since it also forms a sparse data matrix that contains two entries per each pairwise preference in the training set. (Chapelle and Keerthi. 2010) also describe an improved version of the method which they state to have similar scalability as SVM ${ }^{\text {rank }}$, but there is no publicly available implementation of this method.

The experiments are run on a desktop computer with $2.4 \mathrm{GHz}$ Intel Core 2 Duo E6600 processor, 8 GB of main memory, and 64-bit Ubuntu Linux 10.10 operating system. For TreeRSVM, PairRSVM and SVM ${ }^{\text {rank }}$ we use the termination criterion $\epsilon<0.001$, which is the default setting of $\mathrm{SVM}^{\text {rank }}$. The $\epsilon$ parameter has exactly the same meaning and scaling for all of these implementations. For PRSVM we use the termination criterion Newton decrement $<10^{-6}$, as according to Chapelle and Keerthi (2010) this is roughly equivalent 


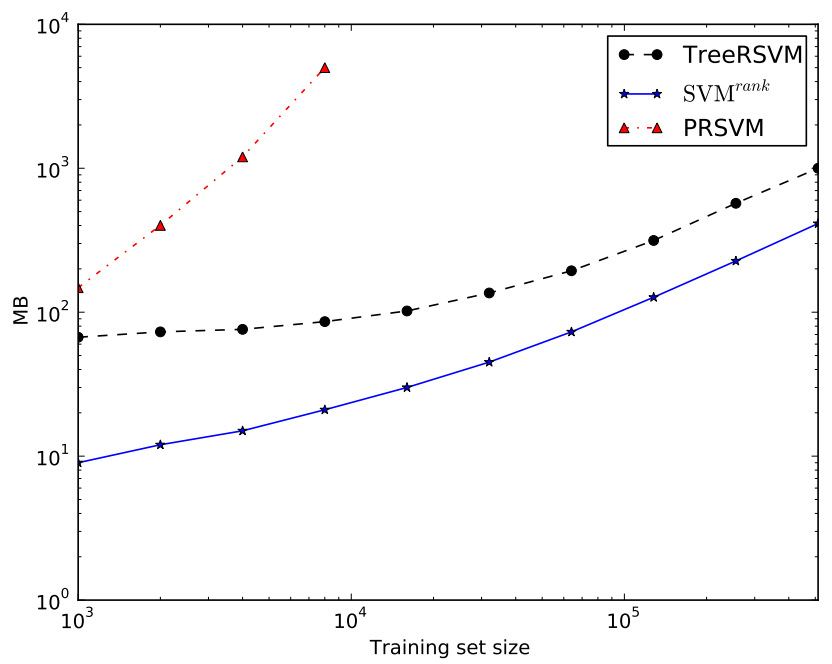

Figure 3: Memory usage for different RankSVM implementations, measured on Reuters.

to the termination criterion we use for the BMRM based methods. SVM ${ }^{\text {rank }}$ and PRSVM use a regularization parameter $C$ that is multiplied to the empirical risk term rather than $\lambda$, and do not normalize the empirical risk by the number of pairwise preferences $N$. Therefore, the proper conversion between the $\lambda$ and $C$ values is $C=\frac{1}{\lambda N}$.

We run experiments of two publicly available data sets. Cadata ${ }^{3}$ is a lowdimensional data set consisting of approximately approximately 20000 examples, each having 8 features. The real valued labels are used directly as utility scores. Our second data set is constructed from the Reuters RCV1 collection (Lewis et al., 2004), and consists of approximately 800000 documents. Here, we use a high dimensional feature representation, with each example having approximately 50000 tf-idf values as features. The data set is sparse, meaning that most features are zero-valued. The utility scores are generated as follows. First, we remove one target example randomly from the data set. Next, we compute the dot products between each example and the target example, and use these as utility scores. In effect, the aim is now to learn to rank documents according to how similar they are to the target document.

Similarly to the scalability experiments of Chapelle and Keerthi (2010), we compute the running times using a fixed value for the regularization parameter, and a sequence of exponentially growing training set sizes. We use $\lambda=10^{-1}$ for Cadata, and $\lambda=10^{-5}$ for Reuters, as these were observed to lead to good test performance. The relative differences in running times between the methods

$\sqrt[3]{\text { http://www.csie.ntu.edu.tw/ cjlin/libsvmtools/datasets/ }}$ 

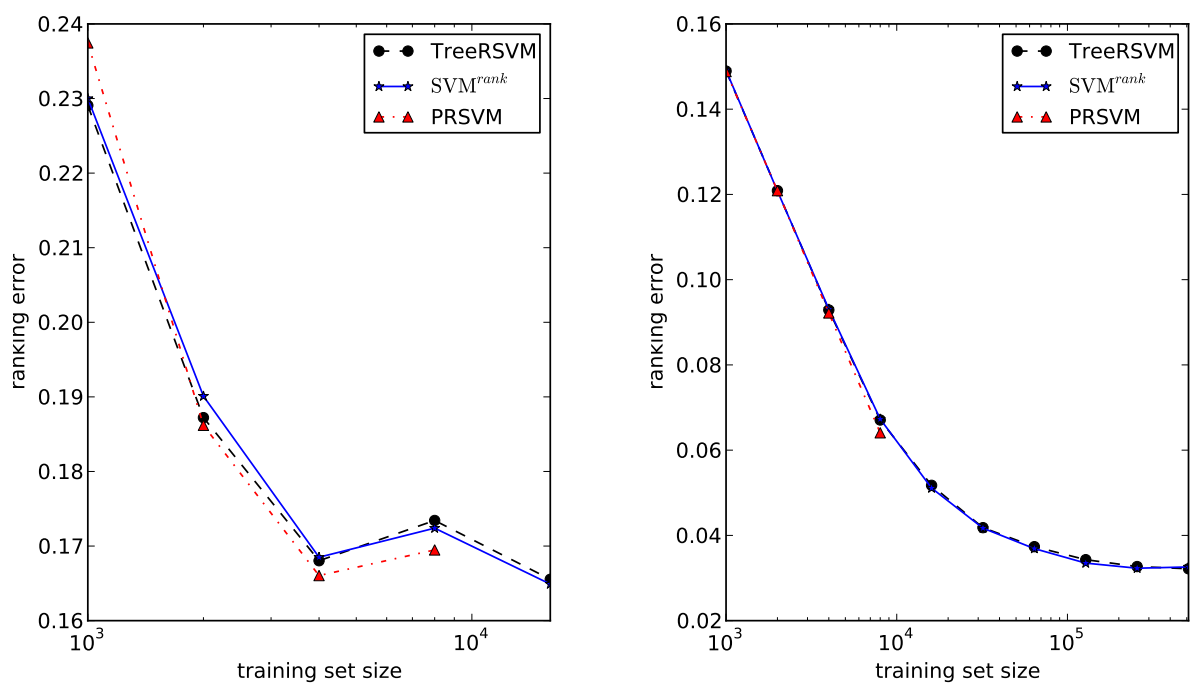

Figure 4: Test error for different RankSVM implementations. Cadata (left) and Reuters (right).

were observed to be similar also for any other tested choices of the regularization parameter values, though the absolute runtimes are for all the methods the larger the smaller the value of $\lambda$ is. For Cadata we consider the training set sizes $[1000,2000,4000,8000,16000]$. For Reuters we consider the training set sizes $[1000,2000,4000,8000,16000,32000,64000,128000,256000,512000]$.

\subsection{Experimental results}

In Figure 1 we plot the average time needed for subgradient computation by the TreeRSVM and the PairRSVM. It can be seen that the results are consistent with the computational complexity analysis, the proposed method scales much better than the one based on iterating over the pairs of training examples in subgradient and loss evaluations. On Reuters with half a million training examples PairRSVM already takes 2760 seconds (46 minutes) to finish a single iteration, whereas the same is achieved by TreeRSVM in 7 seconds.

Next, we compare the scalability of the different RankSVM implementations. In Figure 2 we present the runtimes of all the different implementations, when trained to convergence. As expected, TreeRank achieves orders of magnitude faster training times than the other alternatives. PRSVM could not be trained beyond 8000 examples due to large memory consumption. On Cadata SVM ${ }^{\text {rank }}$ showed much worse scalability than should be expected. More detailed study of the $\mathrm{SVM}^{\text {rank }}$ results revealed that almost all of the runtime was consumed by the quadratic solver, which on some iterations failed to make progress for a 
substantial amount of time. The behavior seemed to be caused by numerical problems, our implementations which use the CVXOPT solver did not have similar issues. On the Reuters data $\mathrm{SVM}^{\text {rank }}$ did not have such problems, the method showed similar scaling as PairRSVM, as expected. With 512000 training examples on Reuters training SVM ${ }^{\text {rank }}$ took 83 hours, and training PairRSVM took 122 hours, whereas training TreeRSVM took only 18 minutes in the same setting.

In Figure 3 we plot the peak memory usage of the considered implementations to give a rough idea about their scalability in terms of memory efficiency. We present results only for the Reuters data, since the Cadata has too few training examples and features per example to allow reliable benchmarking of the methods which have linear scaling in memory usage. PairRSVM is left out of the comparison, since it has almost identical memory consumption as TreeRSVM. PRSVM has quadratic memory complexity with respect to the training set size, and therefore consumes several GB of memory already at 8000 training examples. Both TreeRSVM and SVM ${ }^{\text {rank }}$ start to show linear behavior in memory complexity once the sample size grows large enough, as expected. At first the Python based TreeRSVM implementation is much less memory efficient than the C-language SVM ${ }^{\text {rank }}$ implementation, but as the sample size grows the difference becomes smaller. For the largest sample sizes TreeRSVM uses roughly 2.5 times the amount of memory used by $\mathrm{SVM}^{\text {rank }}$. The difference is due to the fact that the TreeRSVM implementation maintains two copies of the data matrix, one optimized for fast row- and one for fast column access. Better memory efficiency could thus be achieved by maintaining only one copy of data matrix, but initial experiments showed that this would lead to roughly sevenfold increase in training time as measured on Reuters data.

Finally. in Figure 4 we plot the pairwise ranking errors, for the different implementations, as measured on independent test sets. For Cadata we use 4000, and for Reuters 20000 test examples for computing the test errors. PairRSVM is left out of the comparison, since it always reaches exactly the same solution as TreeRSVM. These measurements act as a sanity check, showing that despite the implementational differences TreeRSVM and SVM ${ }^{\text {rank }}$ reach similar performance. Further, we see in the results that even though PRSVM optimizes a squared version of the pairwise hinge loss, it still achieves similar test performance as the other methods.

\section{Conclusion}

In this work we have proposed an $O(m s+m \log (m))$ time method for training RankSVM. Empirical results support the complexity analysis, showing that the method scales well to large data sets. The experiments demonstrate orders of magnitude improvements in training time on large enough data sets, compared to the fastest existing previous implementations. Though we have only considered the linear RankSVM, the approach could also be used to speed up its kernelized version using a reduced set approximation, such as the one proposed 
by Joachims and $\mathrm{Yu}(2009)$. A possible future research direction would be to improve the convergence speed of the BMRM for RankSVM training by devising a line search procedure similar to the one proposed by Franc and Sonnenburg (2009) for SVM classification.

\section{Acknowledgment}

This work has been supported by the Academy of Finland.

\section{References}

Bayer, R., 1972. Symmetric binary B-trees: Data structure and maintenance algorithms. Acta Informatica 1 (4), 290-306.

Bottou, L., Lin, C.-J., 2007. Support vector machine solvers. In: Bottou, L., Chapelle, O., DeCoste, D., Weston, J. (Eds.), Large-Scale Kernel Machines. Neural Information Processing. MIT Press, Cambridge, MA, USA, pp. 1-28.

Bradley, A. P., 1997. The use of the area under the ROC curve in the evaluation of machine learning algorithms. Pattern Recognition 30 (7), 1145-1159.

Burges, C., Shaked, T., Renshaw, E., Lazier, A., Deeds, M., Hamilton, N., Hullender, G., 2005. Learning to rank using gradient descent. In: Raedt, L. D., Wrobel, S. (Eds.), Proceedings of the 22nd international conference on Machine learning (ICML 2005). Vol. 119 of ACM International Conference Proceeding Series. ACM, New York, NY, USA, pp. 89-96.

Chapelle, O., 2007. Training a support vector machine in the primal. Neural Computation 19 (5), 1155-1178.

Chapelle, O., Keerthi, S. S., 2010. Efficient algorithms for ranking with SVMs. Information Retrieval 13 (3), 201-215.

Cormen, T. H., Leiserson, C. E., Rivest, R. L., Stein, C., 2001. Introduction to Algorithms, 2nd Edition. MIT Press, Cambridge, MA, USA.

Cortes, C., Mohri, M., 2004. AUC optimization vs. error rate minimization. In: Thrun, S., Saul, L., Schölkopf, B. (Eds.), Advances in Neural Information Processing Systems 16. MIT Press, Cambridge, MA, USA.

Cortes, C., Vapnik, V., 1995. Support-vector networks. Machine Learning 20 (3), 273-297.

Drucker, H., Burges, C. J., Kaufman, L., Smola, A., Vapnik, V., 1997. Support vector regression machines. In: Mozer, M., Jordan, M., Petsche, T. (Eds.), Advances in Neural Information Processing Systems 9. MIT Press, Cambridge, MA, USA, pp. 155-161. 
Franc, V., Sonnenburg, S., 2009. Optimized cutting plane algorithm for largescale risk minimization. Journal of Machine Learning Research 10, 2157-2192.

Freund, Y., Iyer, R., Schapire, R. E., Singer, Y., 2003. An efficient boosting algorithm for combining preferences. Journal of Machine Learning Research 4, 933-969.

Fürnkranz, J., Hüllermeier, E., 2005. Preference learning. Künstliche Intelligenz $19(1), 60-61$.

Fürnkranz, J., Hüllermeier, E. (Eds.), 2011. Preference Learning, 1st Edition. Springer, Berlin, Heidelberg, Germany.

Hanley, J. A., McNeil, B. J., 1982. The meaning and use of the area under a receiver operating characteristic (ROC) curve. Radiology 143 (1), 29-36.

Herbrich, R., Graepel, T., Obermayer, K., 1999. Support vector learning for ordinal regression. In: Willshaw, D., Murray, A. (Eds.), Proceedings of the Ninth International Conference on Articial Neural Networks (ICANN 1999). Institute of Electrical Engineers, London, pp. 97-102.

Joachims, T., 2002. Optimizing search engines using clickthrough data. In: Hand, D., Keim, D., Ng, R. (Eds.), Proceedings of the 8th ACM SIGKDD Conference on Knowledge Discovery and Data Mining (KDD 2002). ACM Press, New York, NY, USA, pp. 133-142.

Joachims, T., 2005. A support vector method for multivariate performance measures. In: Raedt, L. D., Wrobel, S. (Eds.), Proceedings of the 22nd International Conference on Machine learning (ICML 2005). Vol. 119 of ACM International Conference Proceeding Series. ACM Press, New York, NY, USA, pp. $377-384$.

Joachims, T., 2006. Training linear SVMs in linear time. In: Eliassi-Rad, T., Ungar, L. H., Craven, M., Gunopulos, D. (Eds.), Proceedings of the 12th ACM SIGKDD international conference on Knowledge discovery and data mining (KDD 2006). ACM Press, New York, NY, USA, pp. 217-226.

Joachims, T., Yu, C.-N. J., 2009. Sparse kernel SVMs via cutting-plane training. Machine Learning 76 (2-3), 179-193.

Lewis, D. D., Yang, Y., Rose, T. G., Li, F., 2004. RCV1: A new benchmark collection for text categorization research. Journal of Machine Learning Research 5, 361-397.

Liu, T.-Y., 2009. Learning to rank for information retrieval. Foundations and Trends in Information Retrieval 3 (3), 225-331.

Pahikkala, T., Tsivtsivadze, E., Airola, A., Boberg, J., Järvinen, J., 2009. An efficient algorithm for learning to rank from preference graphs. Machine Learning 75 (1), 129-165. 
Pahikkala, T., Tsivtsivadze, E., Airola, A., Boberg, J., Salakoski, T., 2007. Learning to rank with pairwise regularized least-squares. In: Joachims, T., Li, H., Liu, T.-Y., Zhai, C. (Eds.), SIGIR 2007 Workshop on Learning to Rank for Information Retrieval. pp. 27-33.

Poggio, T., Girosi, F., 1990. Networks for approximation and learning. Proceedings of the IEEE 78 (9).

Provost, F. J., Fawcett, T., Kohavi, R., 1998. The case against accuracy estimation for comparing induction algorithms. In: Shavlik, J. (Ed.), Proceedings of the Fifteenth International Conference on Machine Learning (ICML 1998). Morgan Kaufmann Publishers Inc., San Francisco, CA, USA, pp. 445-453.

Smola, A. J., Vishwanathan, S. V. N., Le, Q., 2007. Bundle methods for machine learning. In: McCallum, A. (Ed.), Advances in Neural Information Processing Systems 20. MIT Press, Cambridge, MA, USA.

Teo, C. H., Smola, A., Vishwanathan, S. V., Le, Q. V., 2007. A scalable modular convex solver for regularized risk minimization. In: Berkhin, P., Caruana, R., Wu, X., Gaffney, S. (Eds.), Proceedings of the 13th ACM SIGKDD international conference on Knowledge discovery and data mining (KDD 2007). ACM, New York, NY, USA, pp. 727-736.

Teo, C. H., Vishwanathan, S. V., Smola, A., Le, Q. V., 2010. Bundle methods for regularized risk minimization. Journal of Machine Learning Research 11, $311-365$.

Tsochantaridis, I., Joachims, T., Hofmann, T., Altun, Y., 2005. Large margin methods for structured and interdependent output variables. Journal of Machine Learning Research 6, 1453-1484.

Waegeman, W., De Baets, B., Boullart, L., 2008. ROC analysis in ordinal regression learning. Pattern Recognition Letters 29 (1), 1-9.

Williams, J. W. J., 1964. Algorithm 232 - Heapsort. Communications of the ACM 7 (6), 347-348. 